\title{
Thrombose des sinus caverneux associée à une sinusite sphénoïdale chez un enfant
}

\author{
Nobuhiko Ohashi MD PhD, Akihiro Otsuka MD, Kanji Yamamoto MD PhD
}

— Citation : CMAJ 2021 May 17; 193:E737. doi : 10.1503/cmaj.201317-f

Voir la version anglaise de l'article ici : www.cmaj.ca/lookup/doi/10.1503/cmaj.201317

$\mathbf{U}$ n garçon de 13 ans s'est présenté à notre centre hospitalier avec des symptômes de fièvre et de maux de tête qui persistaient depuis 1 semaine. Lors de l'examen physique, il était alerte et faisait de la fièvre $\left(40,5^{\circ} \mathrm{C}\right)$. II ne présentait aucune rigidité au cou ni anomalie des mouvements oculaires, de l'apparence des yeux, de l'acuité visuelle ou des sensations faciales. II présentait une numération des leucocytes $(27,2$ [plage normale 3,3$8,6] \times 10^{9} / \mathrm{L}$, avec des neutrophiles segmentés à $85 \%$ ), des protéines $C$ réactives (114,5 [plage normale $<1,5] \mathrm{mg} / \mathrm{L}$ ) et des $\mathrm{D}$-dimères $(28200$ [plage normale $<1000] \mu \mathrm{g} / \mathrm{L}$ ) élevés. L'analyse du liquide céphalorachidien était normale. L'examen d'imagerie par résonance magnétique (IRM) sans agent de contraste montrait une opacification des sinus sphénoïdaux et un examen d'IRM avec agent de contraste montrait des anomalies de remplissage dans les 2 sinus caverneux (figure 1A). Nous avons diagnostiqué une thrombose des sinus caverneux (TSC) secondaire à une sinusite sphénoïdale. L'angiographie par résonance magnétique révélait un rétrécissement des artères carotides internes (figure 2A). Nous avons entrepris l'administration par voie intraveineuse de ceftriaxone, de méropénème et d'héparine et nous avons par la suite isolé la présence d'Haemophilus influenzae dans les hémocultures. Bien que les symptômes du patient se soient améliorés en 1 semaine, sa sinusite sphénoïdale persistait à l'examen d'IRM, menant à une intervention de chirurgie endonasale
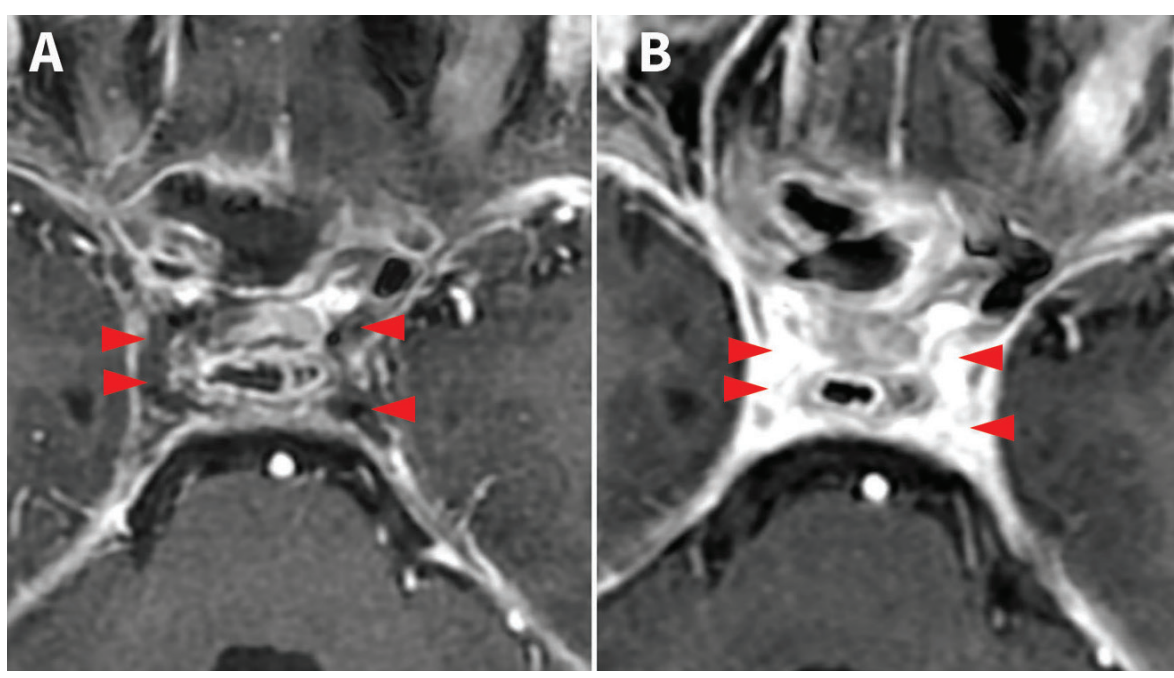

Figure 1 : L'examen d'imagerie par résonance magnétique avec agent de contraste d'un garçon de 13 ans (A) montre des anomalies de remplissage (pointes des flèches) dans les sinus caverneux le premier jour de son hospitalisation, signes de multiples tromboses veineuses qui se sont améliorées au $24^{\mathrm{e}}$ jour (B).
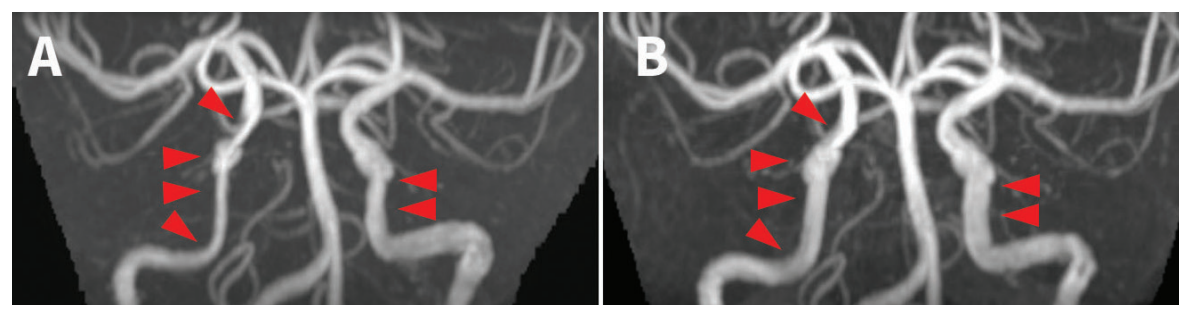

Figure 2 : Images d'angiographie par résonance magnétique d'un garçon de 13 ans montrant un rétrécissement des 2 artères carotides internes (pointes des flèches) au premier jour (A) de l'hospitalisation, rétrécissement qui est disparu au 24e jour de l'hospitalisation (B). 
régionales, comme la sinusite, l'otite moyenne séreuse, la pharyngite ou une infection cutanée du visage. Elle se manifeste le plus souvent par de la fièvre, des maux de tête et des symptômes orbitaux, comme le ptosis et l'ophtalmoplégie $^{1,2}$. L'antibiothérapie est la pierre angulaire du traitement, accompagnée du drainage chirurgical de la collection purulente. Nous avons administré une anticoagulothérapie afin de prévenir le prolongement de la thrombose des sinus caverneux, même si les données probantes soutenant son utilisation sont limitées ${ }^{1,2}$. Le rétrécissement des artères carotides internes est généralement asymptomatique, mais a le potentiel de provoquer des accidents vasculaires cérébraux ischémiques chez les enfants et les adultes ${ }^{1,2}$. Un examen de tomodensitométrie (TDM) ou d'imagerie par résonance magnétique (IRM) avec agent de contraste est recommandé pour diagnostiquer la thrombose des sinus caverneux (TSC) puisque la tomodensitométrie sans agents de contraste ne pourra discerner les anomalies de remplissage spécifiques à la thrombose des sinus caverneux ${ }^{1}$. L'angiographie par résonance magnétique et la tomodensitométrie avec agent de contraste sont utiles pour détecter le rétrécissement des artères carotides internes ${ }^{1,2}$.

\section{Références}

1. Smith DM, Vossough A, Vorona GA, et al. Pediatric cavernous sinus thrombosis: a case series and review of the literature. Neurology 2015;85:763-9.

2. Hsu C-W, Tsai W-C, Lien C-Y, et al. The clinical characteristics, implicated pathogens and therapeutic outcomes of culture-proven septic cavernous sinus thrombosis. J Clin Neurosci 2019;68:111-6.

Intérêts concurrents : Aucun déclaré.

Cet article a été révisé par des pairs.

Les auteurs ont obtenu le consentement parental.

Affiliations : Services de neurologie (Ohashi, Yamamoto) et d'oto-rhino-laryngologie (Otsuka), Hôpital municipal de Nagano, Nagano, Japon.

Propriété intellectuelle du contenu : Il s'agit d'un article en libre accès distribué conformément aux modalités de la licence Creative Commons Attribution (CC BY-NC-ND 4.0), qui permet l'utilisation, la diffusion et la reproduction de tout médium à la condition que la publication originale soit adéquatement citée, que l'utilisation se fasse à des fins non commerciales (c.-à-d., recherche ou éducation) et qu'aucune modification ni adaptation n'y soit apportée. Pour plus de renseignements, consulter le https://creativecommons.org/ licenses/by-nc-nd/4.0/deed.fr.

Remerciements : Les auteurs remercient le Dr Jin Imai pour ses conseils sur l'interprétation radiologique.

Correspondance : Nobuhiko Ohashi, 14mh010c@shinshu-u.ac.jp 